\title{
Protocolo de reabilitação acelerada após reconstrução de ligamento cruzado anterior - dados normativos
}

\section{Accelerated protocol of rehabilitation after anterior cruciate ligament reconstruction with patellar tendon- normative data}

\author{
Edilson Thiele ${ }^{1}$; Luciene Bittencourt ${ }^{2}$; Raul Osiecki ${ }^{2}$; André Montanhol Fornaziero²; Sara Gabellone Hernadez ${ }^{1}$; \\ Paulo Afonso Nunes Nassif ${ }^{3}$; Carmen Marcondes Ribas ${ }^{3}$
}

RES U M O

\begin{abstract}
Objetivo: Avaliar os resultados obtidos com o protocolo de reabilitação acelerada, adaptado às condições de clínica, em pacientes submetidos à operação de reconstrução do ligamento cruzado anterior. Métodos: Foram incluídos 30 pacientes, praticantes de atividade esportiva recreacional, submetidos à operação de reconstrução do ligamento cruzado anterior por meio do tendão patelar. Todos fizeram a reabilitação com o mesmo protocolo de tratamento e no mesmo local. A avaliação isocinética em diferentes ângulos foi realizada antes da operação e no $4^{\circ}$ mês de pós-operatório utilizando dinamômetro isocinético computadorizado da marca Cybex Norm. Resultados: As avaliações no pré-operatório em média demonstraram: pico de torque flexor $93 \%$ a $60 \% \mathrm{~s}$ e $97,3 \%$ a $180 \%$ s; extensor $87,3 \%$ a $60 \%$ e $94,7 \%$ a $180 \%$ s; potência nos músculos flexores de $93,3 \%$ e nos extensores de $96,7 \%$; trabalho muscular dos flexores de $91,7 \%$ e nos extensores de $90,3 \%$; o ângulo do pico de torque flexor de $28,7^{\circ}$, na musculatura extensora o ângulo foi de $62,2^{\circ}$; pico de torque excêntrico nos flexores de $78,3 \%$ e nos extensores de $12,8 \%$. Com quatro meses de pós-operatório os resultados obtidos em média foram: pico de torque flexor $95,4 \%$ a $60 \% \mathrm{~s}$ e $97,1 \% 180 \% \mathrm{~s}$; extensor $70 \%$ a $60 \% \mathrm{~s}$ e $75,7 \%$ a $180 \%$; potência nos músculos flexores de $97,1 \%$ e nos extensores de $79,8 \%$; trabalho muscular dos flexores de $94,2 \%$ e nos extensores de $94,2 \%$; pico de torque excêntrico dos flexores de $84 \%$ e nos extensores de $24,2 \%$; o ângulo do pico de torque flexor foi a $27,3^{\circ}$; na musculatura extensora o ângulo foi de $61,7^{\circ}$. Conclusão: Os resultados demonstraram que os pacientes tratados com o protocolo adaptado apresentam resultados semelhantes aos obtidos com o protocolo original em relação às condições musculares.
\end{abstract}

Descritores: Ligamento cruzado anterior/cirurgia. Joelho. Protocolo de reabilitação acelerada. Avaliação.

\section{INTRODUÇÃO}

O ligamento cruzado anterior (LCA) é responsável por $86 \%$ da restrição do deslocamento anterior da tíbia. A ruptura deste ligamento causa instabilidade crônica do joelho, a qual se não tratada pode evoluir para lesão meniscal, degeneração articular e modificações artríticas'

A lesão deste ligamento é bastante comum no meio esportivo, principalmente nos esportes coletivos, a instabilidade causada pela sua ruptura impossibilita a prática de atividades esportivas que façam o movimento de pivot (giro sobre o próprio eixo).

Dentre as várias técnicas cirúrgicas de reconstrução para recuperar a estabilidade, a operação utilizando o tendão patelar tem a preferência dos cirurgiões, sendo a mais utilizada na atualidade ${ }^{2}$. Nessa técnica cirúrgica o ligamento rompido é substituído pelo 1/3 médio do tendão patelar, sendo fixado em suas extremidades por dois parafusos de interferência. A revascularização deste neoligamento inicia-se na $2^{\text {a }}$ semana e o processo de "ligamentização" prolonga-se até dois anos de pós-operatório ${ }^{3}$.

Em estudo animal observou-se que o neo-ligamento passava por processo de necrose avascular o qual só atingiria a fase de ligamentização aos seis meses estando portanto mais suscetível à rupturas nesta fase ${ }^{4}$. Assim sendo os protocolos de reabilitação eram bastante conservadores em relação ao tempo de recuperação do movimento de extensão do joelho, exercícios com carga, apoio e liberação para atividade esportiva.

Mas os achados de Rougraff et al. ${ }^{3}$, através de análise histológica do neoligamento em humanos, demonstraram que, diferente dos estudos em animais, o enxerto em humanos não apresenta estágio de necrose completa. Existe sim, necrose da porção central do enxerto, porém a porção superficial encontra-se claramente viável, o que é observado pela proliferação celular e a neovascularização proeminente. $\mathrm{O}$ que permite concluir que o enxerto está

Trabalho realizado na Clínica do Joelho, Curitiba, PR, Brasil e Instituto de Pesquisas Médicas da Faculdade Evangélica do Paraná, Curitiba, PR, Brasil.

1. Médicas da Faculdade Evangélica do Paraná, Curitiba, PR, Brasil. 2. Centro de Estudos da Performance Física - CEPEFIS - UFPR. 3. Doutor, Professor Permanente do Programa de Pós-Graduação Em Princípios da Cirurgia e Instituto de Pesquisas Médicas da Faculdade Evangélica do Paraná/Hospital Universitário Evangélico de Curitiba, Curitiba, PR, Brasil. 
parcialmente viável a partir da $3^{\mathrm{a}}$ semana de pós-operatório.

A retirada do 1/3 médio do tendão patelar, a presença de dor, a própria intervenção cirúrgica, entre outros fatores, levam à atrofia muscular pós-operatória, acometendo principalmente o grupo extensor do joelho ${ }^{5}$.

A força do quadríceps apresenta correlação significativa com a estabilidade funcional do joelho, antes e

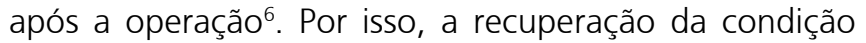
muscular tem sido uma grande preocupação pós-operatória $^{7-10}$.

Muitos protocolos de reabilitação têm sido propostos para evitar as complicações pós-cirúrgicas e recuperar a condição funcional pré-operatória dos pacientes.

Em 1992, Shelbourne e Nitz ${ }^{11}$ faziam a reabilitação de um modo conservador onde no pós-operatório era feita a imobilização por um período de seis a oito semanas. A partir de 1993 o programa de reabilitação foi modificado e a imobilização foi substituída pelo uso imediato do CPM (continuos passive motion). Nos dois anos seguintes o programa de reabilitação sofreu algumas pequenas modificações, pois observou-se que os pacientes mais ousados, e que não respeitaram os prazos determinados pelo programa, evoluíram de maneira mais precoce e recuperaram a função normal do joelho em menor tempo e sem adquirir instabilidade.

Baseados nestas observações Shelbourne e Nitz ${ }^{11}$, fizeram um estudo comparando dois tipos de protocolo de reabilitação, um mais conservador e outro acelerado. O grupo de pacientes submetidos ao programa acelerado recuperou a extensão do joelho mais rapidamente, com isso houve menos casos de complicação onde a perda da extensão levava a nova intervenção cirúrgica. Os exercícios em cadeia cinética fechada utilizados no protocolo acelerado também diminuíram as dores anteriores do joelho e aumentaram a estabilidade subjetiva, além de recuperar a força do quadríceps mais rapidamente. Shelbourne e Gray ${ }^{12}$ também observaram as vantagens da mobilização e o fortalecimento precoce no pós-operatório imediato.

O objetivo deste trabalho foi verificar os benefícios do protocolo de reabilitação acelerada em pacientes submetidos à operação de reconstrução do ligamento cruzado anterior, por meio do tendão patelar, através da avaliação isocinética aplicada no pré-operatório e com quatro meses de pós-operatório.

\section{MÉTODOS}

Após assinatura do termo de consentimento foram avaliados 30 pacientes, praticantes de atividade esportiva recreacional, submetidos à operação de reconstrução do ligamento cruzado anterior por meio do tendão patelar, entre 2002 e 2004 . Todos os pacientes fizeram a reabilitação com o mesmo protocolo de tratamento e no mesmo local. A avaliação isocinética foi realizada antes da cirurgia e no $4^{\circ}$ mês de pós operatório.
Neste protocolo (Tabela 1), a primeira semana teve o objetivo de atingir $90^{\circ}$ de flexão, controlar a dor e o derrame articular. No primeiro mês iniciou-se propriocepção em apoio bipodal com o objetivo de normalização da marcha. A hidroterapia foi incluída a partir da retirada dos pontos e da cicatrização completa dos tecidos. Com dois meses foi realizada a avaliação isocinética utilizando as velocidades angulares de 180 e $240 \%$ s e para aqueles que obtiveram déficit não superior a 35\% iniciaram-se os exercícios com trote em linha reta, evoluindo para trote com mudança de direção e em seguida exercícios com bola. No terceiro mês foi iniciada a musculação com ângulo articular e cargas controladas. Ao final do quarto mês foi feita nova avaliação isocinética, com velocidade angular de 60, 180 e $240 \%$ s no modo concêntrico e $60 \%$ s no modo excêntrico. Manteve-se a musculação e se o déficit muscular do grupo extensor não fosse superior a 35\% na velocidade angular de $60 \%$, iniciava-se o retorno progressivo as atividades esportivas.

Os pacientes avaliados eram do sexo masculino, com idade média de 30,9 +/- 9,4 anos, peso médio 79,3 +/ - 8,8 kg, altura média 1,76 +/- 0,06 m, e índice de massa muscular 25,5+/- 2,2.

Os pacientes foram avaliados em um dinamômetro isocinético computadorizado da marca Cybex Norm (Lumex Inc., Ronkokoma, NY, USA).

A posição de avaliação foi sentado com banco reclinado a $5^{\circ}$, o tronco e coxa firmemente fixadas através de tiras próprias. O eixo de rotação do braço de alavanca do dinamômetro alinhado de maneira visual ao côndilo lateral femoral e o membro inferior fixado ao braço de alavanca do dinamômetro na região maleolar.

As velocidades angulares utilizadas foram de $60 \%$ s, $180 \%$ s e $240 \%$ s no modo concêntrico e $60 \%$ s no modo excêntrico. Os pacientes executaram algumas repetições dos movimentos para a familiarização com o aparelho. Na avaliação propriamente dita, foram feitos cinco movimentos de flexo-extensão do joelho em cada velocidade angular. Ambos os membros foram avaliados e comparados. A avaliação foi precedida de exercício de bicicleta (10 minutos) e exercícios de alongamentos para os músculos avaliados.

\section{RESULTADOS}

Os resultados obtidos na avaliação isocinética são referentes ao membro operado em relação ao membro contralateral não lesionado.

Na tabela 2 estão listados os resultados do pico máximo flexor pré e pós-operatório nas velocidades angulares de $60 \%$ s e $180 \%$ s; da avaliação pré e pós-operatória do pico máximo extensor nas velocidades de $60 \%$ s e $180 \%$ s; da avaliação da potência pré e pós-operatória nos músculos flexores e extensores; do trabalho muscular a $60 /{ }^{\circ} \mathrm{s}$ na melhor repetição executada nos músculos flexores e extensores no pré e pós-operatório; dos ângulos do pico de torque flexor e extensor no pré e no pós-operatório; do 
Tabela 1 - Protocolo de reabilitação adaptado às condições clínicas.

\begin{tabular}{|c|c|}
\hline Objetivos & Tratamento \\
\hline $1^{\text {a }}$ SEMANA & Crioterapia \\
\hline Controle do edema e derrame articular & Exercícios de ADM passiva e ativa para flexão e hiperextensão \\
\hline Controle da dor & Mobilização de patela \\
\hline Controle do quadríceps & Flexo/extensão tornozelo ativa \\
\hline \multirow[t]{5}{*}{$A D M=90$} & Contração isométrica do quadríceps \\
\hline & Exercícios de controle do quadríceps \\
\hline & Mini agachamentos \\
\hline & Heel prop extension \\
\hline & Marcha com 2 muletas \\
\hline
\end{tabular}

\section{$1^{\circ} \mathrm{MÊS}$}

$\mathrm{adM}=0$ a $120^{\circ}$

atividades limitadas

Prevenir derrame articular
Padrão de marcha normal

\author{
$2^{\text {a }}$ semana \\ Propriocepção na bola \\ Elevação da perna com peso \\ Bicicleta \\ Treino de marcha \\ Alongamentos \\ $3^{\mathrm{a}}$ semana \\ Iniciar hidroterapia \\ Esteira \\ $4^{\mathrm{a}}$ semana \\ Propriocepção em apoio bipodal - dynadisc
}

\section{A 8 SEMANAS}

Mobilidade $=$ Membro oposto

Controle de derrame articular

Recuperação da força muscular

\section{MESES}

Déficit de pico de torque $=35 \%$

Avaliação isocinética com velocidade angular $180 \%$ s

\section{MESES}

Recuperar força muscular

Melhora da propriocepção

\section{MESES}

Avaliação isocinética com déficit de força muscular máx. de 25 a

$35 \%$

modo excêntrico nas musculaturas flexora e extensora a $60 \%$ nos períodos pré e pós-operatório. Foram eles no préoperatório: pico de torque flexor $93 \%$ a $60 \%$ s e $97,3 \%$ a $180 \%$ s; extensor $87,3 \%$ a $60 \%$ s e $94,7 \%$ a $180 \%$ s; potência nos músculos flexores de 93,3\% e nos extensores de 96,7\%; trabalho muscular dos flexores de $91,7 \%$ e nos extensores de 90,3\%; o ângulo do pico de torque flexor de $28,7^{\circ}$, na musculatura extensora o ângulo foi de $62,2^{\circ}$; pico de torque excêntrico nos flexores de 78,3\% e nos extensores de $12,8 \%$. Com quatro meses de pós-operatório os resultados obtidos foram: pico de torque flexor $95,4 \%$ a $60 \%$ s e $97,1 \%$ $180 \%$ s; extensor $70 \%$ a $60 \%$ s e $75,7 \%$ a $180 \%$ s; potência
Propriocepção em apoio monopodal

Exercícios extensão terminal - CCA

Exercícios em cadeia cinética fechada
Intensificar exercícios de força e alongamentos

Iniciar trote em linha reta

Trote com mudança de direção

Exercícios com bola

Iniciar musculação

Intensificar exercícios proprioceptivos

Intensificar exercícios específicos do esporte praticado 
ser complicação perigosa e limitante no retorno as atividades esportivas pré-lesão. A avaliação isocinética tem sido considerada um teste confiável, com o coeficiente de correlação intraclass, de 0,98 - 0,99 para velocidades de 60 e $180 \% \mathrm{~s}^{13}$. Por isso avaliou-se a performance isocinética do quadríceps e dos ísquios tibiais em pacientes submetidos a reconstrução ligamentar tratados com o protocolo acelerado.

Shelbourne et al. ${ }^{14}$, relataram recuperação da perfomance da musculatura extensora de $70 \%$ após 13 semanas da operação com o tendão patelar na velocidade de $180 \%$ s. Porém, em seu estudo parte dos pacientes avaliados foram reabilitados com o protocolo acelerado e parte com o protocolo tradicional. Carlo et al. ${ }^{15}$, fizeram um estudo comparativo entre a recuperação com o protocolo acelerado e o tradicional, demonstrando que com o tradicional a recuperação dos ísquios tibiais aos 3 , 6 e 12 meses de pós-operatório foi de 79,41, 90,99 e $95,13 \%$ respectivamente, enquanto que os pacientes reabilitados com o protocolo acelerado apresentaram $92,65,97,76$ e $98,73 \%$. Na musculatura extensora os pacientes do protocolo tradicional apresentaram recuperação de $63,94,71,48$ e $80,02 \%$, enquanto que no protocolo acelerado os resultados foram $69,63,76,81$ e $87,42 \%$.

Rosberg et al. ${ }^{16}$, avaliou pacientes submetidos à reconstrução ligamentar com o tendão patelar após 12 a 24 meses de evolução e constatou $18 \%$ de perda de força em relação ao membro contralateral do quadríceps na velocidade angular de $60 \% \mathrm{~s}$, e de $10 \%$ nos ísquios tíbiais. Porém a $180 \%$ s nenhum teve perda em nenhum dos grupos musculares avaliados.

Natri et al. ${ }^{17}$, compararam a performance isocinética em pacientes crônicos e agudos pós reconstrução de LCA. As avaliações foram feitas em média quatro anos após o procedimento cirúrgico utilizando as velocidades angulares de 60 e $180 \%$ s para pico de torque e $180 \% \mathrm{~s}$ para trabalho máximo. O grupo crônico apresentou déficit no pico de torque de quadríceps de $20 \%$ e o agudo de
$15 \%$ a $60 \%$ s e de 9 e $18 \%$ nos grupos agudo e crônico respectivamente na velocidade angular de $180 \%$ s. Os ísquios tibiais apresentaram déficit de 7 e $8 \%$ nos grupos agudo e crônico na velocidade angular de $60 \%$ s e 26 e 8 \% na velocidade angular de $180 \%$ s.

Risberg et al. ${ }^{18}$, fizeram um estudo prospectivo em pacientes com reconstrução ligamentar, demonstrando que aos seis meses de pós-operatório a recuperação do trabalho total, avaliado a $60 \%$ nos músculos extensores foi de $66,4 \%$, com 12 meses foi de $81,6 \%$ e com dois anos de $92,6 \%$. Nos músculos flexores aos seis meses a recuperação foi de $83,9 \%$, aos 12 meses foi de $93,1 \%$ e aos dois anos de $95,1 \%$.

Neste estudo a recuperação do trabalho total aos quatro meses de pós-operatório foi em média de 94,2\% nos músculos flexores e de $74,1 \%$ nos músculos extensores.

Carter e Edinger ${ }^{19}$, compararam a recuperação pós-operatória nas reconstruções ligamentares com tendão flexor e o patelar com seis meses da operação. Os grupos foram avaliados na velocidade angular de $180 \%$ s obtendo os seguintes resultados nos músculos extensores: tendão patelar $68,3 \%$, semitendíneo/grácil 78,1\% e semitendíneo $74,3 \%$. Nos músculos flexores foi observado: tendão patelar $86,1 \%$, semitendíneo/grácil 81,7\% e semitendíneo 80,6\%

A comparação dos resultados entre os diversos trabalhos deve ser feita de maneira cuidadosa, pois é necessário que os parâmetros utilizados na avaliação sejam semelhantes. Portanto os estudos de Shelbourne ${ }^{16}$, Carlo et al. ${ }^{2}$ e Carter e Edinger ${ }^{3}$, apresentam condições semeIhantes a este trabalho, tendo sido utilizada a mesma velocidade angular $\left(180^{\circ} / \mathrm{s}\right)$, mas o período de avaliação pósoperatória apresenta pequena variação. Mas utilizando estes estudos em comparação aos resultados aqui apresentados, verificou-se que são semelhantes em relação ao pico de torque em ambos os grupos musculares.

Em conclusão, os resultados demonstraram que os pacientes tratados com o protocolo adaptado apresentam resultados semelhantes aos obtidos com o protocolo original em relação às condições musculares.

Tabela 2 - Média e desvio-padrão dos resultados do pré e pós-operatório (Teste t).

\begin{tabular}{lccc}
\hline & Pré-operatório & Pós-operatório & p \\
\hline Pico máximo flexor a 60\%s (\%) & 97,03 & 95,45 & 0,5173 \\
Pico máximo flexor a 180\%s (\%) & 97,31 & 97,10 & 0,9521 \\
Pico máximo extensor a 60\%s (\%) & 87,31 & 70,00 & $0,0001^{*}$ \\
Pico máximo extensor a 180\%s (\%) & 97,79 & 75,76 & $0,0001^{*}$ \\
Potência Muscular flexora (\%) & 93,03 & 27,20 & 0,5098 \\
Trabalho muscular flexor a 60\%s (\%) & 91,79 & 94,24 & 0,5360 \\
Trabalho muscular extensor a 60\%(\%) & 90,31 & 74,14 & 0,5360 \\
Ângulo de pico de torque flexor & $28,72^{\circ}$ & $27,48^{\circ}$ & 0,4300 \\
Ângulo de pico de torque extensor & $62,28^{\circ}$ & $61,72^{\circ}$ & 0,8169 \\
Pico de torque flexor no modo excêntrico a 60\%s (\%) & 78,31 & 84,07 & 0,2484 \\
Pico de torque extensor no modo excêntrico a 60\% (\%) & 87,14 & 75,72 & 0,0105 \\
\hline
\end{tabular}


Objective: evaluate the gotten results of the accelerated protocol adapted to the clinic conditions in CLA post operatory patients. Methods: 30 patients were selected and submitted to an isokynetic test at the pre operatory and 4 months post operatory. Results: the isokinetics evaluations at the pre operatory presented: flexor peak torque $93 \%$ at $60 \%$ and $97,3 \%$ at $180 \%$. Extensor $87,3 \%$ at $60 \%$ and $94,7 \%$ at $180 \%$; power of the flexor muscles of $93,3 \%$ and extensors of $96,7 \%$; the muscular work of the flexors was of $91,7 \%$ and extensors of $90,3 \%$; the flexor peak torque angle was at $28,7^{\circ}$. At the extensor musculature the angle was at $62,2^{\circ}$; flexors eccentric peak torque of $78,3 \%$ and the extensors of $12,8 \%$. With 4 months of post operatory the gotten results showed: flexor peak torque $95,4 \%$ at $60 \%$ and $97,1 \%$. at $180 \%$; extensor $70 \%$ at $60 \% \mathrm{~s}$ and $75,7 \%$. at $180 \%$; power of the flexor muscles of $97,1 \%$ and extensors of $79,8 \%$; the muscular work of the flexors was of $94,2 \%$ and extensors of $94,2 \%$; flexors eccentric peak torque of $84 \%$ and extensors of $24,2 \%$; the flexor peak torque angle was at 27,3\%; in extensor musculature the angle was at $61,7^{\circ}$. Conclusion: showed that the patients treated with the adapted protocol presented similar results to the original protocol in relation to the muscular conditions.

Key words: Anterior cruciate ligament/surgery. Knee. Accelereted protocol of rehabilitation. Evaluation.

\section{REFERÊNCIAS}

1. Butler DL, Noyes FR, Grood ES. Ligamentous restraints to anterior, posterior drawer in the human knee. A biomechanical study. J.Bone Joint Surg Am. 1980; 62(2):259-70.

2. Penteado PC, Marchetto A, Nunes Neto JF, Pereira PP. Tratamento cirúrgico das lesões do ligamento cruzado anterior. Rev Joelho. 2003; 3(1):19-24

3. Rourgraff B, Shelbourne KD, Gerth PK, Warner J. Arhroscopic and histologic analysis of human patellar tendon autografts used for anterior cruciate ligament reconstruction. Am. J Sports Med. 1993; 21(2):277-84

4. O'Connor JJ, Zavatsky A. Anterior cruciate ligament function in the normal knee. In: Jackson DW, Arnoczky SP, Frank CB, Woo SLY, Simon TM. The anterior cruciate ligament - Current and future concepts. New York: Raven Press; 1993. p. 39-52.

5. Shelbourne DK, Patel DV. Prevention of complications after autogenous bone-patellar tendon-bone $\mathrm{ACL}$ reconstruction. Instr Course Lect. 1996; 45: 253-62.

6. Keays SL, Bullock-Saxton JE, Newcombe P, Keays AC. The relationship between Knee strength and functional stability before and after anterior cruciate ligament reconstruction. J Orthop Res. 2003; 21(2):231-7.

7. Fitzgerald GK, Piva SR, Irrgang JJ. A modified neuromuscular eletrical stimulation protocol for quadriceps strength training following anterior cruciate ligament reconstruction. J Orthop Sports Phys Ther. 2003; 33(9):492-501.

8. Morrissey MC, Brewster C, Shields CL Jr, Brown M. The effects of electrical stimulation on the quadriceps during postoperative knee immobilization. Am J Sports Med. 1985; 13(1):40-5.

9. Snyder-Mackler L, Delitto A, Bailey SL, Stralka SW. Strength of the quadriceps femoris muscle and functional recovery after reconstruction of anterior cruciate ligament. A prospective, randomized clinical trial of electrical stimulation. J Bone Joint Surg Am. 1995; 77(8):1166-73.

10. Tyler T.F, Nicholas SJ, Hershman EB, Glace BW, Mullaney MJ, Mchugl MP. The effect of creatine supplementation on strength recovery after anterior cruciate ligament $(\mathrm{ACL})$ reconstruction: a randomized, placebo-controlled, double-blind-trial. Am J Sports Med. 2004; 32(2):383-8.

11. Shelbourne KD, Nitz P. Accelerated rehabilitation after anterior cruciate ligament reconstruction. J Orthop Sports Phys Ther. 1992; 15(6):256-64.

12. Shelbourne KD, Gray T. Anterior cruciate ligament reconstruction with autogenous patellar tendon graft followed by accelerated rehabilitation. A two to nine year follow up. Am J Sports Med. 1997: 25(6):786-95

13. Levene JA, Hart BA, Seeds RH, Fuhrman GA. Reliability of reciprocal isokinetic testing of the knee extensors and flexors. J Orthop Sports Phys Ther. 1991; 4(3):121-7

14. Shelbourne KD, Wilckens JH, Mollbashy A, Carlo MS. Artrofibrosis in acute anterior cruciate ligament reconstruction. The effect of timing of reconstruction and rehabilitation. Am J Sports Med. 1991; 19(4):332-6.

15. Carlo MS, Shelbourne KD, Mccarrol JR, Retting AC. Traditional versus ccelerated Rehabilitation following $A C L$ reconstruction: $A$ one year follow-up. J Orthop Sports Phys Ther. 1992; 15(6):30916.

16. Rosenberg TD, Franklin JL, Baldwin NG, Nelson KA. Extensor mechanism function after patellar tendon graft harvest for anterior cruciate ligament reconstruction. Am J Sports Med. 1992; 20(5):519-25.

17. Natria $A$, Järvinem $M$, Latvala $K$, Kannus $P$. Isokinetic muscle performance after anterior cruciate ligament surgery. Long-term results and outcome predicting factors after primary surgery and late-phase reconstruction. Int J Sports Med. 1996; 17(3):223-8.

18. Risberg MA, Holm I, Tjomsland O, Ljunggren E, Ekeland A. Prospective study of changes in impairments and disabilities after anterior cruciate ligament reconstruction. J Orthop Sports Phys Ther.1999; 29(7):400-12.

19. Carter ES, Edinger S. Isokinetic evaluation of anterior cruciate ligament reconstruction: hamstring versus patellar tendon. Arthroscopy. 1999; 15(2):169-72.

Recebido em 04/12/2008

Aceito para publicação em 05/02/2009

Conflito de interesse: nenhum

Fonte de financiamento: nenhuma

\section{Como citar este artigo:}

Thiele E, Bittencourt L, Osiecki R, Fornaziero AM, Hernadez SG, Nassif PAN, Ribas CM. Protocolo de reabilitação acelerada após reconstrução de ligamento cruzado anterior - dados normativos. Rev Col Bras Cir. [periódico na Internet] 2009; 36(6). Disponível em URL: http:// www.scielo.br/rcbc

\section{Endereço para correspondência:}

Edilson Thiele

E-mail: Edilson.thiele@terra.com.br 